\title{
Mechanism of Resistance to Dietary Cholesterol
}

\author{
Lindsey R. Boone, ${ }^{1,2}$ Patricia A. Brooks, ${ }^{2}$ Melissa I. Niesen, ${ }^{2}$ and Gene C. Ness ${ }^{2}$ \\ ${ }^{1}$ Department of Pathology, McGowan Institute for Regenerative Medicine, University of Pittsburgh, Pittsburgh, PA 15219, USA \\ ${ }^{2}$ Department of Molecular Medicine, College of Medicine, University of South Florida, Tampa, FL 33612, USA
}

Correspondence should be addressed to Gene C. Ness, gness@hsc.usf.edu

Received 25 May 2011; Revised 27 July 2011; Accepted 28 July 2011

Academic Editor: Akihiro Inazu

Copyright (C) 2011 Lindsey R. Boone et al. This is an open access article distributed under the Creative Commons Attribution License, which permits unrestricted use, distribution, and reproduction in any medium, provided the original work is properly cited.

Background. Alterations in expression of hepatic genes that could contribute to resistance to dietary cholesterol were investigated in Sprague-Dawley rats, which are known to be resistant to the serum cholesterol raising action of dietary cholesterol. Methods. Microarray analysis was used to provide a comprehensive analysis of changes in hepatic gene expression in rats in response to dietary cholesterol. Changes were confirmed by RT-PCR analysis. Western blotting was employed to measure changes in hepatic cholesterol $7 \alpha$ hydroxylase protein. Results. Of the 28,000 genes examined using the Affymetrix rat microarray, relatively few were significantly altered. As expected, decreases were observed for several genes that encode enzymes of the cholesterol biosynthetic pathway. The largest decreases were seen for squalene epoxidase and lanosterol $14 \alpha$ demethylase (CYP 51A1). These changes were confirmed by quantitative RT-PCR. LDL receptor expression was not altered by dietary cholesterol. Critically, the expression of cholesterol $7 \alpha$ hydroxylase, which catalyzes the rate-limiting step in bile acid synthesis, was increased over 4 -fold in livers of rats fed diets containing $1 \%$ cholesterol. In contrast, mice, which are not resistant to dietary cholesterol, exhibited lower hepatic cholesterol $7 \alpha$ hydroxylase (CYP7A1) protein levels, which were not increased in response to diets containing $2 \%$ cholesterol.

\section{Introduction}

There is considerable variation among animals and humans in terms of their responses to consumption of excess dietary cholesterol. Consumption of a high-cholesterol diet does not automatically result in elevated serum cholesterol levels due to the operation of adaptive responses. Rabbits, hamsters, and $\mathrm{C} 57 \mathrm{BL} / 6$ mice are not resistant to dietary cholesterol and exhibit marked elevations in serum cholesterol levels when given diets supplemented with cholesterol [1-3]. On the other hand, rats such as Sprague-Dawley, Wistar-Furth, Spontaneously Hypertensive or Fischer 344 show very little if any increase in serum cholesterol levels when given a similar cholesterol challenge [2]. For most humans, consumption of increased amounts of dietary cholesterol produces only small increases in both LDL and HDL cholesterol with little effect on the ratio of LDL to $\mathrm{HDL}[4,5]$. In a 14 -year study of over 80,000 female nurses, egg consumption was unrelated to the risk of coronary heart disease [4]. On balance, extensive epidemiologic studies show that dietary cholesterol is not a contributor to increased heart disease risk in humans
[6]. Clearly, in many rat strains and most people, adaptive responses are operating that keep serum cholesterol levels within the normal range.

Multiple possible mechanisms may be operating to provide a person or animal with resistance to the serum cholesterol-raising action of dietary cholesterol. These include decreasing the rate of cholesterol biosynthesis, decreasing the rate of cholesterol absorption, increasing the rate of cholesterol excretion, increasing the conversion of cholesterol to bile acids, and increasing the rate of removal of serum cholesterol via liver lipoprotein receptors $[7,8]$.

In order to obtain a comprehensive and unbiased analysis of adaptive responses in hepatic gene expression to dietary cholesterol, we carried out microarray analysis of changes in Sprague-Dawley rat liver gene expression elicited by a $1 \%$ cholesterol diet. These animals are known to have adaptive responses that render them resistant to dietary cholesterol. In order to induce atherosclerotic plaques in these animals, the rats must be rendered hypothyroid [9]. Thus, this animal is a reasonable model for human responses to dietary cholesterol. In a previous microarray study, C57BL/6 mice were used 
[10]. These mice become hypercholesterolemic and develop fatty lesions in their ascending aortas when given diets supplemented with cholesterol $[11,12]$.

Liver was selected for extensive study, because this tissue not only synthesizes cholesterol but also is also responsible for bile acid production and excretion of cholesterol and expresses the majority of the body's LDL receptors [13].

\section{Methods}

2.1. Animals. Male Sprague-Dawley rats, $150-200 \mathrm{~g}$ (Harlan, Madison, Wis, USA), were fed Harlan Teklad 22/5 rodent chow with or without $1 \%$ cholesterol for five days. The animals were kept in a reversed lighting cycle room (12 dark $/ 12 \mathrm{hrs}$ light) and sacrificed at $0900-1000 \mathrm{hrs}$, corresponding to the third to fourth hour of the dark period when cholesterol biosynthesis is maximal. Twelve-week old C57BL/6J mice were fed chow with or without $2 \%$ cholesterol for 5 days. Liver samples were obtained, and microsomes were prepared from both rats and mice [14]. All procedures were carried out according to the regulations and oversight of the University of South Florida Institutional Animal Care and Use Committee (IACUC), protocol 2953.

2.2. Cholesterol Analysis. Trunk blood was collected and allowed to clot. The samples were centrifuged at 5,000 $\mathrm{xg}$ for $5 \mathrm{~min}$. The serum was removed with a Pasteur pipette. Total serum cholesterol levels were determined by a cholesterol oxidase method using Infinity Cholesterol Reagent (Sigma) [15]. Values are expressed as $\mathrm{mg} / \mathrm{dL}$ of serum. Liver cholesterol levels were determined as previously described [16]. Briefly, weighed liver samples were saponified, extracted with petroleum ether and subjected to reverse-phase highperformance liquid chromatography on a Spheri-5, RP18, 5 reverse-phase column (Altech Associates). Values are expressed as $\mathrm{mg} / \mathrm{g}$ of liver.

2.3. RNA Isolation. A portion of about $200 \mathrm{mg}$ was quickly excised from livers of the rats and immediately homogenized in $4 \mathrm{~mL}$ of Tri-Reagent from Molecular Research Center (Cincinnati, Ohio, USA) using a Polytron homogenizer at room temperature. The remainder of the isolation steps was carried out using volumes corresponding to $4 \mathrm{x}$ the manufacturer's recommendations. RNA concentrations were determined by diluting each sample $1: 100$ and measuring its absorbance at $260 \mathrm{~nm}$.

2.4. Microarray Analysis. Isolated RNA was further purified using the RNeasy kit from Qiagen. To demonstrate that the RNA was indeed free of RNase activity, samples were incubated in water at $42^{\circ} \mathrm{C}$ for $1 \mathrm{hr}$ and then examined on $1 \%$ agarose gels. An identical pattern of bands in unheated and heated samples was obtained showing a lack of RNase activity. Microarray analysis was performed by the Moffitt Core Facility (Tampa, FL) using the Affymetrix GeneChip Instrument system and the protocol established by Affymetrix, Inc. Ten $\mu \mathrm{g}$ of RNA each from the livers of 3 control and 3 cholesterol-fed rats was used in the analysis. The RNA was converted to double-stranded cDNA using an oligo(dT)24 primer containing a T7 RNA polymerase recognition sequence. The product was transcribed into biotin-labeled cRNA using T7 RNA polymerase. The biotinylated cRNA was hybridized to Affymetrix GeneChip Rat Genome 230 Plus 2.0 arrays, which detects about 28,000 genes. Multiple oligos were used for each gene with the data averaged. Scanned chip images were analyzed using GeneChip algorithms.

2.5. Real Time RT-PCR Analysis. To validate the microarray results, we assessed the expression of a subset of genes via real-time PCR essentially as described previously [14]. Total RNA was resuspended in diethyl pyrocarbonate-treated water. Twenty micrograms of RNA was then DNAse-treated using the TURBO DNA-Free Kit from Ambion. cDNA was prepared from $1 \mu \mathrm{g}$ of DNAse-treated RNA using the Reverse Transcription System from Promega. The final product was brought up to $100 \mu \mathrm{L}$, and a total of $2 \mu \mathrm{L}$ of the reverse transcription reaction was then used for real-time PCR analysis. The primer sequences used are given in Table 1. PCR was carried out according to the protocol from ABI Power SYBR Green Master Mix, using a Chromo-4 DNA Engine (Bio-Rad) with minor modifications. The program used for amplification was (i) $95^{\circ} \mathrm{C}$ for 5 minutes, (ii) $95^{\circ} \mathrm{C}$ for $15 \mathrm{sec}-$ onds, (iii) $61^{\circ} \mathrm{C}$ for 1 minute (collect data), and (iv) go to step (ii) 40 times, (v) $55^{\circ} \mathrm{C}+0.5^{\circ} \mathrm{C}$ each 10 seconds, $\times 80$ (melt curve). The results were quantified by the $\Delta \Delta \mathrm{C}_{\mathrm{t}}$ method using Microsoft Excel statistical programs and SigmaPlot 8.0. As a housekeeping gene, $18 \mathrm{~S}$ ribosomal RNA was used. Values are expressed relative to this.

2.6. Western Blot Analysis. At time of sacrifice, a portion of liver was excised for protein analysis. Lysosome-free liver microsomes were prepared according to the procedure previously described [17]. Liver microsomes $(50 \mu \mathrm{g}$ of protein per lane) from rats and mice were subjected to SDS-PAGE and western blotting. Membranes were incubated overnight with $1: 2,000$ dilution of cholesterol $7 \alpha$ hydroxylase primary antibody generated in rabbits and generously provided to us by Dr. Mats Rudling in 5\% PBST nonfat dry milk. A $1: 10,000$ dilution of Sheep anti Rabbit IGG was as the secondary antibody. The West Pico Chemiluminesence kit was used for detection with exposure times ranging from 5 to 20 seconds. The blots were then stripped and reprobed with an antibody to $\beta$-actin. The resulted were expressed relative to the $\beta$-actin signal.

2.7. Statistics. For the microarray data, a 2-tailed distribution $t$-test, equal variance was used. The RT-PCR data are presented as means \pm standard errors. $P$ values using a 2 -tailed distribution $t$-test are given.

\section{Results}

3.1. Microarray Analysis. In order to comprehensively identify the genes that exhibit significant alterations in rates of transcription in response to dietary cholesterol, microarray 
TABLE 1: Oligonucleotide sequences used for RT-PCR analysis.

\begin{tabular}{|c|c|c|}
\hline Gene & Primers & Sequence $\left(5^{\prime} \rightarrow 3^{\prime}\right)$ \\
\hline \multirow{2}{*}{ SQLE } & Sense & AGTGAACAAACGAGGCGTCCTACT \\
\hline & Antisense & AAAGCGACTGTCATTCCTCCACCA \\
\hline \multirow{2}{*}{ CYP51 } & Sense & TTAGGTGACAACCTGACACACGCT \\
\hline & Antisense & TGCTTACTGTCTTGCTCCTGGTGT \\
\hline \multirow{2}{*}{ ABCG8 } & Sense & GATGCTGGCTATCATAGGGAGC \\
\hline & Antisense & TCTCTGCCTGTGATAACGTCGA \\
\hline \multirow{2}{*}{ ABCG5 } & Sense & TGAGCTCTTCCACCACTTCGACAA \\
\hline & Antisense & TGTCCACCGATGTCAAGTCCATGT \\
\hline \multirow{2}{*}{ ACAT2 } & Sense & TTGTGCCAGTGCACGTGTCTTCTA \\
\hline & Antisense & GCTTCAGCTTGCTCATGGCTTCAA \\
\hline \multirow{2}{*}{ CYP7A } & Sense & TGAAAGCGGGAAAGCAAAGACCAC \\
\hline & Antisense & TCTTGGACGGCAAAGAGTCTTCCA \\
\hline \multirow{2}{*}{ 7DHCR } & Sense & TCAGCTTCCAGGTGCTGCTTTACT \\
\hline & Antisense & ACAATCCCTGCTGGAGTTATGGCA \\
\hline \multirow{2}{*}{ D14SR } & Sense & AATGGTTTCCAGGCTCTGGTGCTA \\
\hline & Antisense & ATAAAGCTGGTGAGAGTGGTCGCA \\
\hline \multirow{2}{*}{ HMGCR } & Sense & ATTGCACCGACAAGAAACCTGCTG \\
\hline & Antisense & TTCTCTCACCACCTTGGCTGGAAT \\
\hline \multirow{2}{*}{ HMGCS } & Sense & TTGGTAGTTGCAGGAGACATCGCT \\
\hline & Antisense & AGCATTTGGCCCAATTAGCAGAGC \\
\hline \multirow{2}{*}{ IGFBP1 } & Sense & AGAGGAACAGCTGCTGGATAGCTT \\
\hline & Antisense & AGGGCTCCTTCCATTTCTTGAGGT \\
\hline \multirow{2}{*}{ LANS } & Sense & ACTCTACGATGCTGTGGCTGTGTT \\
\hline & Antisense & AAATACCCGCCACGCTTAGTCTCA \\
\hline \multirow{2}{*}{ LIPIN2 } & Sense & TCTGCCATGGACTTGCCTGATGTA \\
\hline & Antisense & ACTCGTGGTACGTGATGATGTGCT \\
\hline \multirow{2}{*}{$\operatorname{PPAR} \alpha$} & Sense & AGACCTTGTGCATGGCTGAGAAGA \\
\hline & Antisense & AATCGGACCTCTGCCTCCTTGTTT \\
\hline \multirow{2}{*}{$\operatorname{PPAR} \gamma$} & Sense & CAATGCCATCAGGTTTGGGCGAAT \\
\hline & Antisense & ATACAAATGCTTTGCCAGGGCTCG \\
\hline \multirow{2}{*}{ SC4MEOX } & Sense & ACCTGGCACTATTTCCTGCACAGA \\
\hline & Antisense & AGCCTGGAACTCGTGATGGACTTC \\
\hline
\end{tabular}

TABLE 2: Serum and liver cholesterol levels.

\begin{tabular}{lcc}
\hline Condition & Serum & Liver \\
\hline & $\mathrm{Mg} / 100 \mathrm{~mL}$ & $\mathrm{Mg} / \mathrm{g}$ \\
Normal & $128 \pm 5$ & $3.2 \pm 0.3$ \\
Cholesterol fed & $102 \pm 4$ & $6.2 \pm 0.5^{*}$ \\
\hline
\end{tabular}

${ }^{*} P<0.01$ compared with normal chow fed rats. A 2-tailed distribution $t$ test, equal variance was used.

analysis was performed on hepatic RNA from SpragueDawley rats fed either normal rodent chow or chow supplemented with $1 \%$ cholesterol for 5 days. This dietary regimen does not raise serum cholesterol levels in these male Sprague Dawley rats $[2,18]$. Liver cholesterol levels are increased about 2 -fold (Table 2). This is similar to our previous findings with $2 \%$ cholesterol [16]. Thus, this treatment appears to constitute a useful model for studying adaptive responses.
Surprisingly, the rates of transcription of relatively few genes were altered more than 2 -fold (Table 3 ). The largest increase was observed for insulin-like growth factor-binding protein 1 . This increase of over 4 -fold was confirmed by RT-PCR analysis (Table 5). A prominent increase of nearly 3 -fold in hepatic cholesterol $7 \alpha$ hydroxylase expression was observed. This would provide for increased production of bile acids and more efficient elimination of biliary cholesterol, since this enzyme catalyzes the rate-limiting step of bile acid synthesis. ABCG5 and ABCG8 expression was actually decreased (Table 4). These ATP-binding cassette (ABC) transport proteins promote biliary secretion of neutral sterols [19]. Also, acetyl CoA acetyltransferase 2 (ACAT2), which esterifies excess cholesterol, was significantly decreased. Strikingly, there was no change in hepatic LDL receptor expression.

Many of the genes exhibiting downregulated expression in response to dietary cholesterol (Table 3) catalyze reactions of the cholesterol biosynthetic pathway. Levels of mRNA for the enzyme that catalyzes the rate-limiting reaction, HMGCoA reductase, were only decreased 2-fold. RT-PCR analysis (Table 4) showed only a 1.4-fold decrease HMG-CoA reductase, in agreement with previous results from Northern blotting analysis [20]. The largest decrease (7.9-fold) was observed for squalene epoxidase. This enzyme regulates lanosterol synthesis [21]. The next largest decrease among cholesterol biosynthetic enzymes was seen for lanosterol $14 \alpha$ demethylase. The expression of several cholesterol biosynthetic enzymes including $\Delta 14$ sterol reductase, sterol C4 methyl oxidase-like, 7-dehydrocholesterol reductase, HMG-CoA synthase 1, farnesyl diphosphate synthase, lanosterol synthase, acetoacetyl CoA synthase, and mevalonate kinase were decreased 2 to 4 -fold (Table 4). Other cholesterol biosynthetic enzymes such as sterol-C5-desaturase, phosphomevalonate kinase, diphosphomevalonate decarboxylase, 24-dehydrocholesterol reductase and dehydrogenase/reductase (SDR family) member 7 were decreased 1.5- to 1.9-fold. Other genes of interest whose expression was decreased by dietary cholesterol were SREBP-2 (1.4-fold, $P=0.028)$ and PCSK9 (1.5-fold, $P=0.022)$.

3.2. RT-PCR Analysis. Quantitative RT-PCR was utilized to examine a subset of the altered genes that were identified via microarray analysis. The results are presented in Table 5 and correlate with those seen in the microarray analysis. For example, the expression of squalene epoxidase (Sqle) and lanosterol $14 \alpha$ demethylase (Cyp51A1) were decreased by 8.29 and 4.53-fold, respectively (Table 5) as compared with 7.9 and 3.5-fold in the microarray analysis (Table 4). The relative fold changes in ABCG5 and 8, HMG-CoA synthase, ACAT-2, lanosterol synthase, sterol C4 methyl oxidase-like, lipin 2, cholesterol $7 \alpha$ hydroxylase, and 7-dehydrocholesterol reductase also agree very closely, providing further verification of the data obtained from the microarray analysis.

3.3. Western Blotting Analysis. Since the increase in rat liver cholesterol $7 \alpha$ hydroxylase mRNA caused by dietary cholesterol could be important for the elimination of cholesterol 
TABLe 3: Microarray analysis identifying genes upregulated by dietary cholesterol.

\begin{tabular}{|c|c|c|c|c|c|c|c|}
\hline & Normal & & & holester & & Fold diff & Gene \\
\hline 386 & 507 & 439 & 2377 & 1264 & 2347 & $+4.5^{* *}$ & Insulin-like growth factor binding protein 1 \\
\hline 476 & 457 & 536 & 2322 & 2418 & 988 & $+3.9^{*}$ & B-cell leukemia/lymphoma 6 (predicted) \\
\hline 3597 & 5311 & 8493 & 16287 & 17914 & 12394 & $+2.7^{* *}$ & Cholesterol $7 \alpha$ hydroxylase \\
\hline 73 & 304 & 546 & 853 & 747 & 746 & $+2.5^{*}$ & Similar to Ran-binding protein 2 (predicted) \\
\hline 2287 & 1733 & 1841 & 5590 & 5726 & 5166 & $+2.8^{* *}$ & Zinc finger protein $354 \mathrm{~A}$ \\
\hline 127 & 387 & 320 & 756 & 494 & 773 & $+2.4^{*}$ & $\operatorname{PPAR} \alpha$ \\
\hline 253 & 372 & 250 & 766 & 759 & 536 & $+2.4^{* *}$ & Lipin 2 (predicted) \\
\hline 34 & 131 & 263 & 332 & 303 & 344 & $+2.3^{*}$ & Zinc finger, matrin-like (predicted) \\
\hline 207 & 684 & 856 & 1358 & 1006 & 1277 & $+2.1^{*}$ & HGPRTase \\
\hline 4255 & 2830 & 4209 & 9654 & 4959 & 8308 & +2.0 & Glucose-6-phosphatase \\
\hline
\end{tabular}

${ }^{*} P<0.05 ;{ }^{* *} P<0.01$. A 2 -tailed distribution $t$-test, equal variance was used.

TABLE 4: Microarray analysis identifying genes downregulated by dietary cholesterol.

\begin{tabular}{|c|c|c|c|c|c|c|c|}
\hline \multicolumn{3}{|c|}{ Normal } & \multicolumn{3}{|c|}{ Cholesterol } & \multirow{2}{*}{$\frac{\text { Fold diff }}{-7.9^{* *}}$} & \multirow{2}{*}{$\begin{array}{l}\text { Gene } \\
\text { Squalene epoxidase }\end{array}$} \\
\hline 1185 & 2381 & 1598 & 219 & 232 & 203 & & \\
\hline 588 & 783 & 426 & 148 & 84 & 143 & $-4.8^{* *}$ & ABCG8 \\
\hline 574 & 373 & 195 & 51 & 144 & 62 & -4.5 & Hypo protein XP_580018 \\
\hline 2353 & 3066 & 2285 & 735 & 783 & 503 & $-3.8^{*}$ & Acetyl CoA Acetyltransferase 2 \\
\hline 10377 & 11676 & 10752 & 2690 & 3350 & 3329 & $-3.5^{* *}$ & Lanosterol $14 \alpha$ demethylase \\
\hline 3890 & 4874 & 3668 & 929 & 1280 & 1426 & $-3.4^{* *}$ & Delta 14 sterol reductase \\
\hline 1049 & 893 & 1196 & 186 & 216 & 542 & $-3.3^{* *}$ & Cytokine inducible $\mathrm{SH} 2$ protein \\
\hline 238 & 316 & 242 & 65 & 115 & 76 & $-3.1^{* *}$ & Nuc factor, erythroid derived 2 \\
\hline 733 & 1023 & 760 & 233 & 232 & 345 & $-3.1^{* *}$ & Ephrin A5 \\
\hline 1998 & 608 & 524 & 340 & 343 & 375 & -3.0 & Dual specificity phosphatase1 \\
\hline 11250 & 14322 & 9729 & 3511 & 4636 & 4315 & $-2.8^{* *}$ & Sterol C4 methyl oxidase-like \\
\hline 6083 & 6326 & 4997 & 1961 & 2597 & 2103 & $-2.6^{* *}$ & 7-Dehydrocholesterol reductase \\
\hline 2851 & 2771 & 2112 & 1027 & 879 & 1055 & $-2.6^{* *}$ & Solute carrier family 25 mem 30 \\
\hline 14079 & 17666 & 16412 & 5555 & 6296 & 7154 & $-2.5^{* *}$ & HMG-CoA Synthase 1 \\
\hline 14890 & 13208 & 12147 & 5146 & 5920 & 5178 & $-2.5^{* *}$ & Farnesyl diphosphate synthase \\
\hline 1857 & 2636 & 1792 & 882 & 1025 & 635 & $-2.5^{* *}$ & Lanosterol synthase \\
\hline 4946 & 5502 & 4267 & 1503 & 2456 & 1667 & $-2.6^{* *}$ & Farnesyl diphos transferase 1 \\
\hline 1695 & 2518 & 1567 & 870 & 834 & 814 & $-2.3^{*}$ & ABCG5 \\
\hline 13476 & 13702 & 14018 & 6048 & 7357 & 5541 & $-2.2^{* *}$ & Fatty acid desaturase 1 \\
\hline 10599 & 8161 & 6620 & 3050 & 4215 & 4797 & $-2.1^{*}$ & Hemato expressed homeobox \\
\hline 444 & 591 & 470 & 225 & 288 & 205 & $-2.1^{* *}$ & Acetoacetyl CoA synthase \\
\hline 1269 & 1465 & 1136 & 604 & 749 & 548 & $-2.0^{* *}$ & Hypo protein XP_579849 \\
\hline 10087 & 11135 & 8727 & 4223 & 5304 & 5287 & $-2.0^{* *}$ & HMGCR \\
\hline 2516 & 2177 & 2744 & 1138 & 1049 & 1509 & $-2.0^{* *}$ & Forkhead box A2 \\
\hline 848 & 1080 & 873 & 538 & 444 & 432 & $-2.0^{* *}$ & Mevalonate kinase \\
\hline
\end{tabular}

${ }^{*} P<0.05$; ${ }^{* *} P<0.01$. A 2 -tailed distribution $t$-test, equal variance was used.

from the body, we wished to determine whether levels of this protein are actually increased. For comparison purposes, we examined the effect of dietary cholesterol on mouse liver cholesterol $7 \alpha$ hydroxylase protein. Cholesterol $7 \alpha$ hydroxylase protein levels were much higher in rat liver than in mouse liver (Figure 1). Supplementing the chow with $1 \%$ cholesterol increased hepatic cholesterol $7 \alpha$ hydroxylase protein levels in rats from $1.22 \pm 0.78$ to $6.52 \pm 2.27$
(4 chow-fed rats compared with 5 cholesterol-fed animals with a $P=0.011$ ). In contrast, little effect on the levels in mouse liver was seen even when $2 \%$ cholesterol was added to the diet (Figure 1).

3.4. Time-Course Experiment. A time-course experiment was conducted to determine how rapidly dietary cholesterol reduces the expression of Sqle and Cyp51. RT-PCR analysis, 
TABLE 5: RT-PCR analysis.

\begin{tabular}{|c|c|c|c|c|}
\hline Gene & Normal & Cholesterol & Fold & $P$ value \\
\hline ABCG5 & $1.00 \pm 0.07$ & $0.67 \pm 0.09$ & -1.49 & 0.018 \\
\hline ABCG8 & $1.02 \pm 0.17$ & $0.17 \pm 0.03$ & -6.00 & 0.003 \\
\hline HMG-CoA synthase & $1.03 \pm 0.23$ & $0.23 \pm 0.01$ & -4.48 & 0.008 \\
\hline Squalene epoxidase & $1.17 \pm 0.26$ & $0.14 \pm 0.03$ & -8.29 & 0.002 \\
\hline Lanosterol $14 \alpha$ demeth & $0.71 \pm 0.28$ & $0.16 \pm 0.01$ & -4.53 & 0.027 \\
\hline Delta 14 sterol reductase & $1.03 \pm 0.31$ & $0.19 \pm 0.15$ & -5.33 & 0.013 \\
\hline Lanosterol synthase & $1.12 \pm 0.58$ & $0.33 \pm 0.14$ & -3.39 & 0.083 \\
\hline ACAT-2 & $1.00 \pm 0.08$ & $0.31 \pm 0.07$ & -3.20 & 0.001 \\
\hline Sterol C4 Me Ox like & $1.04 \pm 0.31$ & $0.39 \pm 0.50$ & -2.65 & 0.128 \\
\hline 7-Dehydrocholesterol red & $1.01 \pm 0.13$ & $0.50 \pm 0.06$ & -2.00 & 0.004 \\
\hline HMG-CoA reductase & $1.02 \pm 0.26$ & $0.73 \pm 0.28$ & -1.40 & 0.261 \\
\hline PPAR gamma & $1.07 \pm 0.40$ & $1.02 \pm 0.89$ & -1.04 & 0.944 \\
\hline PPAR alpha & $1.01 \pm 0.18$ & $1.06 \pm 0.44$ & +1.05 & 0.852 \\
\hline Lipin 2 (predicted) & $1.03 \pm 0.29$ & $1.64 \pm 0.42$ & +1.59 & 0.105 \\
\hline Chol $7 \alpha$ hydroxylase & $1.17 \pm 0.35$ & $3.27 \pm 0.97$ & +2.79 & 0.044 \\
\hline IGFBP1 & $1.02 \pm 0.31$ & $4.30 \pm 1.90$ & +4.22 & 0.042 \\
\hline
\end{tabular}

A 2-tailed distribution $t$-test, equal variance was used.

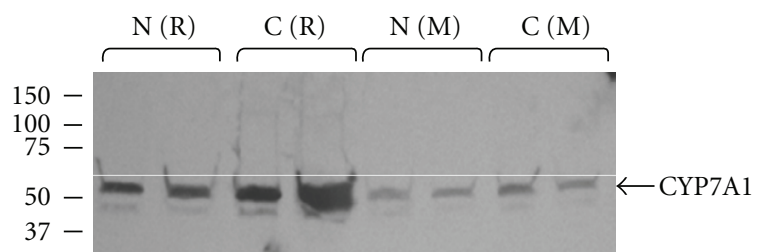

FIGURE 1: Comparison of the effects of dietary cholesterol on hepatic cholesterol $7 \alpha$ hydroxylase protein levels in rats and Mice. Rats (R) and mice (M) were fed normal (N) chow diets with or without $1 \%$ or $2 \%$ cholesterol (C) for 5 days. A representative Western blot of hepatic microsomal cholesterol $7 \alpha$ hydroxylase protein is presented.

as shown in Figures 2 and 3, demonstrates that the decrease in Sqle occurred more rapidly. Both Sqle and Cyp51 decreased progressively over a 3 -day period. This agrees with the time course for the reduction in translation of HMGCoA reductase mRNA [22].

\section{Conclusion}

Sprague Dawley rats from Harlan fed the grain-based Tekland 22/5 rodent chow diet resist a high cholesterol dietinduced increase in serum cholesterol as reported previously $[2,16]$. They do show increases in liver cholesterol levels (Table 2). This indicates that dietary cholesterol is effectively taken up by the intestine, where Niemann-Pick C1-like 1 protein facilitates its uptake [23]. The resulting chylomicron remanents are taken up by the liver. With the increased cholesterol from the diet, the activity of the hepatic LDL receptor, as determined from its rate of turnover, is markedly decreased [18]. Thus, the cycling of this receptor, located in the cholesterol-rich caveolae portion of the plasma

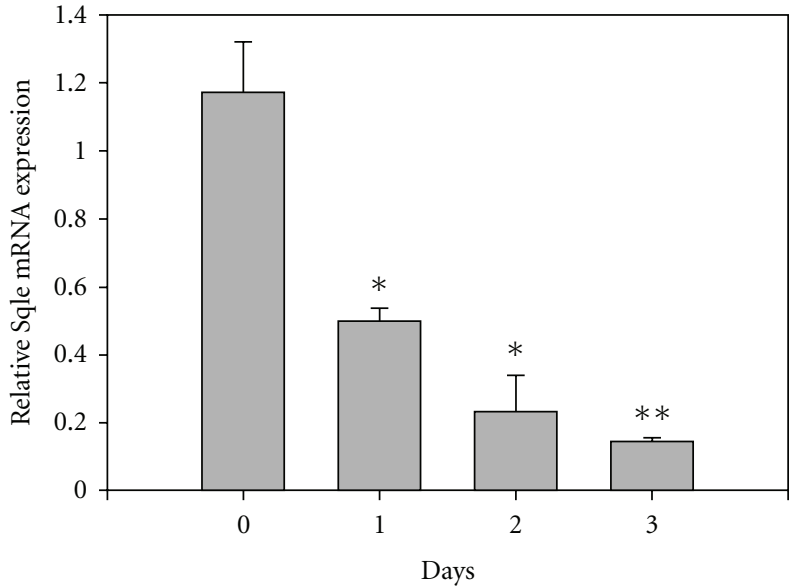

FIGURE 2: Effect of cholesterol feeding on hepatic squalene epoxidase mRNA levels. Rats were fed a diet containing 1\% cholesterol for the indicated number of days. Hepatic mRNA levels were determined by RT-PCR. Values are given as means \pm Standard Error for 3 to 6 rats per time point. ${ }^{*} P<0.05 ;{ }^{*} P<0.01$.

membrane [24], virtually stops [18]. The levels of hepatic LDL receptor protein and mRNA do not, however, change [18]. Since the liver must play a key role in adapting to and resisting the increase load of cholesterol, we conducted a microarray study to investigate changes in hepatic mRNA levels.

Of all the hepatic genes examined, the most dramatic changes seen in response to dietary cholesterol were the decreases in Sqle and Cyp51. These changes seen in the microarray experiment were confirmed by RT-PCR. When lanosterol levels are decreased as occurs when Sqle expression is decreased, the different $\mathrm{Kms}$ of the three reactions catalyzed 


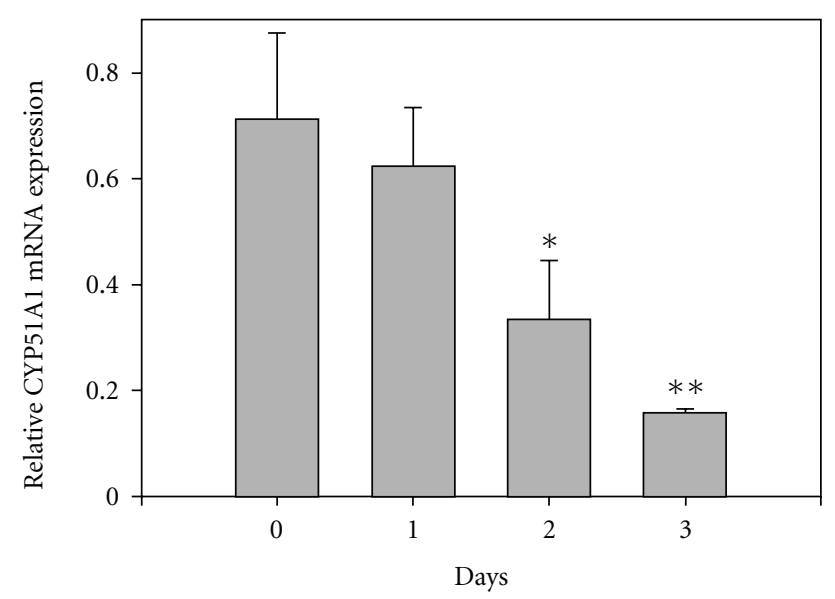

FIgURE 3: Effect of cholesterol feeding on hepatic lanosterol $14 \alpha$ demethylase mRNA levels. Rats were fed a diet containing $1 \%$ cholesterol for the indicated number of days. Hepatic mRNA levels were determined by RT-PCR. Values are given as means \pm Standard Error for 3 to 6 rats per time point. ${ }^{*} P<0.05$; ${ }^{* *} P<0.01$.

by Cyp 51 come into play. The $\mathrm{Km}$ for the formation of $3 \beta$ hydroxylanosterol-8-en-32-al is $56 \mu \mathrm{M}$ while the $\mathrm{Km}$ for its destruction (conversion to 4 dimethylcholesta 8, 14 dien$3 \beta-\mathrm{ol})$ is $368 \mu \mathrm{M}$ [25]. Although there would be sufficient lanosterol for the first reaction catalyzed by CYP51 (formation of $3 \beta$-hydroxylanosterol-8-en-32-al), because of the low $\mathrm{Km}$, its subsequent conversion to 4 dimethylcholesta 8,14 dien- $3 \beta$-ol would be slow due to the high $\mathrm{Km}$ for this reaction. Thus, $3 \beta$-hydroxylanosterol-8-en-32-al would accumulate. This oxylanosterol is known to act to decrease the rate of translation of hepatic HMG-CoA reductase in $\mathrm{CHO}$ cells [26]. The structural analogue, $15 \alpha$-fluoro-3 $\beta$ hydroxylanost-7-en-32-aldehyde has also been reported to inhibit translation of HMG-CoA reductase mRNA in CHO cells [27]. In addition, it has been demonstrated that feeding the nonmetabolizable oxylanosterol analogue, 15 oxa-32vinyllanost-8-ene-3 $\beta$, 32 diol to rats results in a marked decrease in translation of hepatic HMG-CoA reductase mRNA which mimics the effect of dietary cholesterol [28]. Thus, dietary cholesterol-mediated decreases in Sqle and Cyp51 transcription lead to decreased translation of hepatic HMGCoA reductase mRNA.

In this microarray study, a 2-fold decrease in HMG-CoA reductase expression was observed in response to dietary cholesterol. The SREs in the promoters of both Sqle and Cyp51 $[29,30]$ have been shown to be responsive to SREBP2 and would be expected to be downregulated by cholesterol feeding. However, the SRE sequence in the HMG-CoA reductase promoter does not agree well with the consensus SRE, which may explain the only modest decrease in HMG-CoA reductase transcription in response to dietary cholesterol. Previous Northern blotting analysis and nuclear run-on studies showed only slight decreases in mRNA levels and rates of transcription for hepatic HMG-CoA reductase [20, 31]. However, enzyme activity and protein levels drop to a couple percent of control values $[20,31]$. This is due primarily to a marked decrease in the rate of translation of HMG-CoA reductase mRNA caused by feeding cholesterol [22]. The decrease in the rate of translation of HMG-CoA reductase mRNA is due to shift from association of the mRNA with polysomes to monosomes caused by dietary cholesterol [32]. The present observations are predicted by the proposed two-step model for feedback regulation of hepatic HMG-CoA reductase gene expression [33]. In this model, increased hepatic cholesterol leads to decreased formation of mature SREBP2 and decreased transcription of Sqle and Cyp51. This in turn results in accumulation of $3 \beta$ hydroxylanosterol-8-en-32-al, which acts to decrease translation of hepatic HMG-CoA reductase mRNA [20].

In addition to adapting to excess dietary cholesterol by decreasing the rate of hepatic cholesterol biosynthesis, adaptations in bile acid pathways also occurred. A 2.7-fold increase was observed in cholesterol $7 \alpha$ hydroxylase expression, which is the enzyme that catalyzes the rate-limiting step in bile acid synthesis (Table 3 ). We have previously observed by Northern blotting analysis that hepatic cholesterol $7 \alpha$ hydroxylase mRNA is markedly increased in rats [16]. Paradoxically, the microarray analysis did not show increases in expression of the ABCG5/G8 transporters. Instead, decreases in the expression of both ABCG5 and ABCG8 were observed. The expression of ABCG8, in particular, was markedly decreased. These findings were confirmed by RT-PCR analysis (Table 4). This is in contrast to the study in mice where ABCG5 expression was reported to be upregulated about 2fold in response to dietary cholesterol [10]. In agreement with previous Northern blotting analysis, cholesterol feeding did not affect hepatic LDL receptor mRNA levels [18]. However, the rate of cycling and hence activity of the receptor is markedly decreased in response to dietary cholesterol [18].

Western blotting analysis showed that dietary cholesterol increases hepatic cholesterol $7 \alpha$ hydroxylase protein levels in rats but not in mice (Figure 1). Also, mice exhibited lower levels of cholesterol $7 \alpha$ hydroxylase protein in their livers. This is in agreement with a previous study [34] showing that dietary cholesterol caused a 3-fold increase in hepatic cholesterol $7 \alpha$ hydroxylase mRNA in rats with much less effect in C57BL/6J mice. Other reports showed that dietary cholesterol may act to increase or decrease cholesterol $7 \alpha$ hydroxylase mRNA levels in C57BL/6J mice depending on the type of fat added to the diet $[35,36]$. Diets supplemented with olive oil (monounsaturated fatty acids) caused increased expression of hepatic cholesterol $7 \alpha$ hydroxylase. Western blotting analysis of cholesterol $7 \alpha$ hydroxylase was not preformed in these previous studies.

In a recent study comparing the responses of hamsters and rats to cholesterol-enriched diets, it was found that hepatic cholesterol $7 \alpha$ hydroxylase expression in hamsters was not increased in response to cholesterol feeding [37]. This is in contrast with the increase seen in rats. However, hamsters do not adapt well to cholesterol supplemented diets, as their serum cholesterol levels are increased about 2fold in response to a $0.1 \%$ cholesterol diet [37]. Also, hamsters express much lower (only 2 percent of that of rats) basal levels of hepatic HMG-CoA reductase [2], which renders them less able to resist the effects of dietary cholesterol [13]. 
The effect of a high cholesterol diet on the expression of genes in livers of mice has been previously investigated by microarray analysis [10]. In that study, Sqle was found to be downregulated 3.4 and 2.8-fold in males and females, respectively. This correlates with the decrease (7.9-fold) observed for hepatic Sqle expression in the present study. Apparently, no changes in CYP51 or cholesterol $7 \alpha$ hydroxylase expression were observed in the previous study [10]. HMG-CoA reductase was decreased 2-fold in males with no effect seen in females [10]. The greatest change in the previous study was observed for a disintegrin and metalloprotease domain 11 family member (Adam 11) which was upregulated 5 to 11 -fold in the microarray and TaqMan analysis. ADAM 11 is a specific aggrecanase induced by interleukin 6 [38] which would promote an inflammatory response. In the present analysis, no change in Adam 11 expression was observed. A significant increase in serum amyloid A3, an acute phase reactant, was also observed in the earlier study [10]. An increase in this protein also indicates an inflammatory response to a high cholesterol diet.

The largest cholesterol-induced increase in hepatic gene expression in the present study was observed for insulinlike growth factor-binding protein 1 (IGFBP1), which was measured at over 4 -fold by both microarray and RT-PCR analysis. This protein is produced by the liver during inflammation [39], possibly caused by dietary cholesterol. Increased levels are associated with an elevated risk of cardiovascular disease in type 2 diabetic patients [40]. IGFBP1 may also be a marker for insulin resistance [41] and be involved in the development of type 2 diabetes [42]. It may be protective against the growth of certain cancers by binding insulin-like growth factor-1, a potent mitogen [43].

In summary, a comprehensive examination of the adaptive hepatic transcriptional responses to dietary cholesterol was conducted using microarray and RT-PCR analysis. The data suggest that rats adapt, in part, to excess dietary cholesterol by markedly reducing hepatic cholesterol biosynthesis and enhancing elimination of cholesterol as bile acids due to induction of cholesterol $7 \alpha$ hydroxylase. A large increase in insulin-like growth factor-binding protein 1 was also observed. This could partially explain the inflammatory response associated with excess dietary cholesterol.

\section{Abbreviations}

SQLE: Squalene epoxidase

CYP51: Lanosterol $14 \alpha$ demethylase

ABCG8: ATP binding cassette G8

ABCG5: ATP binding cassette G5

ACAT2: Acetyl CoA Acetyltransferase 2

CYP7A: Cholesterol $7 \alpha$ hydroxylase

7DHCR: 7-Dehydrocholesterol reductase

D14SR: Delta 14 sterol reductase

HMGCR: 3-Hydroxy-3-methylglutaryl coenzyme A reductase

HMGCS: 3-Hydroxy-3-methylglutaryl coenzyme A synthase

IGFBP1: Insulin-like growth factor binding protein 1

LANS: Lanosterol synthase
PPAR $\alpha$ : Peroxisome proliferator activated receptor $\alpha$

PPAR $\gamma$ : Peroxisome proliferator activated receptor $\gamma$

SCMEOX: Sterol C4 methyl oxidase-like.

\section{Authors' Contributions}

L. R. Boone, P. A. Brooks, M. I. Niesen, and G. C. Ness carried out experiments. L. R. Boone and G. C. Ness designed the experiments. L. R. Boone and G. C. Ness wrote the paper. All authors read and approved the final paper.

\section{Conflict of Interests}

The authors declare that they have no conflict of interests.

\section{Acknowledgments}

This research was supported by Grants no. 04 TSP-03 from the Florida Department of Health and no. R01 DK 075414 from NIH. The authors are most grateful to Dr. Mats Rudling, Karolinska Institutet, Stockholm, Sweden, for his generous gift of cholesterol $7 \alpha$ hydroxylase antibody.

\section{References}

[1] S. B. Biddinger, K. Almind, M. Miyazaki, E. Kokkotou, J. M. Ntambi, and C. R. Kahn, "Effects of diet and genetic background on sterol regulatory element-binding protein-1c, linebreak stearoyl-CoA desaturase 1, and the development of the metabolic syndrome," Diabetes, vol. 54, no. 5, pp. 13141323, 2005.

[2] G. C. Ness and K. R. Gertz, "Hepatic HMG-CoA reductase expression and resistance to dietary cholesterol," Experimental Biology and Medicine, vol. 229, no. 5, pp. 412-416, 2004.

[3] K. Prasad, "Regression of hypercholesterolemic atherosclerosis in rabbits by secoisolariciresinol diglucoside isolated from flaxseed," Atherosclerosis, vol. 197, no. 1, pp. 34-42, 2008.

[4] F. B. Hu, M. J. Stampfer, J. E. Manson et al., "Dietary fat intake and the risk of coronary heart disease in women," The New England Journal of Medicine, vol. 337, no. 21, pp. 1491-1499, 1997.

[5] D. J. McNamara, "Dietary cholesterol and atherosclerosis," Biochimica et Biophysica Acta, vol. 1529, no. 1-3, pp. 310-320, 2000.

[6] D. J. McNamara, M. B. Katan, R. M. Weggemans, and P. L. Zock, "Eggs and heart disease risk: perpetuating the misperception," The American Journal of Clinical Nutrition, vol. 75, no. 2, pp. 333-335, 2002.

[7] D. W. Bilheimer, J. L. Goldstein, and S. M. Grundy, "Liver transplantation to provide low-density-lipoprotein receptors and lower plasma cholesterol in a child with homozygous familial hypercholesterolemia," The New England Journal of Medicine, vol. 311, no. 26, pp. 1658-1664, 1984.

[8] C. Gälman, Y. Bonde, M. Matasconi, B. Angelin, and M. Rudling, "Dramatically increased intestinal absorption of cholesterol following hypophysectomy is normalized by thyroid hormone," Gastroenterology, vol. 134, no. 4, pp. 11271136, 2008.

[9] T. G. Cole, I. Kuisk, W. Patsch, and G. Schonfeld, "Effects of high cholesterol diets on rat plasma lipoproteins and 
lipoprotein-cell interactions," Journal of Lipid Research, vol. 25, no. 6, pp. 593-603, 1984.

[10] K. N. Maxwell, R. E. Soccio, E. M. Duncan, E. Sehayek, and J. L. Breslow, "Novel putative SREBP and LXR target genes identified by microarray analysis in liver of cholesterol-fed mice," Journal of Lipid Research, vol. 44, no. 11, pp. 2109-2119, 2003.

[11] S. A. Schreyer, D. L. Wilson, and R. C. Leboeuf, "C57BL/6 mice fed high fat diets as models for diabetes-accelerated atherosclerosis," Atherosclerosis, vol. 136, no. 1, pp. 17-24, 1998.

[12] J. L. Stewart-Phillips and J. Lough, "Pathology of atherosclerosis in cholesterol-fed, susceptible mice," Atherosclerosis, vol. 90, no. 2-3, pp. 211-218, 1991.

[13] G. C. Ness and C. M. Chambers, "Feedback and hormonal regulation of hepatic 3-hydroxy-3-methylglutaryl coenzyme A reductase: the concept of cholesterol buffering capacity (44508)," Experimental Biology and Medicine, vol. 224, no. 1, pp. 8-19, 2000.

[14] W. R. Lagor, R. Heller, E. D. De Groh, and G. C. Ness, "Functional analysis of the hepatic HMG-CoA reductase promoter by in vivo electroporation," Experimental Biology and Medicine, vol. 232, no. 3, pp. 353-361, 2007.

[15] G. C. Ness, R. C. Holland, and D. Lopez, "Selective compensatory induction of hepatic HMG-CoA reductase in response to inhibition of cholesterol absorption," Experimental Biology and Medicine, vol. 231, no. 5, pp. 559-565, 2006.

[16] D. Lopez and G. C. Ness, "Inhibitors of 3-hydroxy-3-methylglutaryl coenzyme A reductase unmask transcriptional regulation of hepatic low-density lipoprotein receptor gene expression by dietary cholesterol," Archives of Biochemistry and Biophysics, vol. 344, no. 1, pp. 215-219, 1997.

[17] G. C. Ness, C. E. Sample, and M. Smith, "Characteristics of rat liver microsomal 3-hydroxy-3-methylglutaryl coenzyme A reductase," Biochemical Journal, vol. 233, no. 1, pp. 167-172, 1986.

[18] G. C. Ness, Z. Zhao, and D. Lopez, "Inhibitors of cholesterol biosynthesis increase hepatic low-density lipoprotein receptor protein degradation," Archives of Biochemistry and Biophysics, vol. 325, no. 2, pp. 242-248, 1996.

[19] D. M. Small, "Role of ABC transporters in secretion of cholesterol from liver into bile," Proceedings of the National Academy of Sciences of the United States of America, vol. 100, no. 1, pp. 4-6, 2003.

[20] G. C. Ness, R. K. Keller, and L. C. Pendleton, "Feedback regulation of hepatic 3-hydroxy-3-methylglutaryl-CoA reductase activity by dietary cholesterol is not due to altered mRNA levels," Journal of Biological Chemistry, vol. 266, no. 23, pp. 14854-14857, 1991.

[21] J. Sakakibara, R. Watanabe, Y. Kanai, and T. Ono, "Molecular cloning and expression of rat squalene epoxidase," Journal of Biological Chemistry, vol. 270, no. 1, pp. 17-20, 1995.

[22] C. M. Chambers and G. C. Ness, "Dietary cholesterol regulates hepatic 3-hydroxy-3-methylglutaryl coenzyme A reductase gene expression in rats primarily at the level of translation," Archives of Biochemistry and Biophysics, vol. 354, no. 2, pp. 317-322, 1998.

[23] M. A. Valasek, J. J. Repa, G. Quan, J. M. Dietschy, and S. D. Turley, "Inhibiting intestinal NPC1L1 activity prevents diet-induced increase in biliary cholesterol in Golden Syrian hamsters," The American Journal of Physiology, vol. 295, no. 4, pp. G813-G822, 2008.

[24] G. C. Ness, N. Kohlruss, and K. R. Gertz, "Association of the low-density lipoprotein receptor with caveloae in hamster and rat liver," Biochemical and Biophysical Research Communications, vol. 303, pp. 177-181, 2003.

[25] J. M. Trzaskos, R. T. Fischer, and M. F. Favata, "Mechanistic studies of lanosterol C-32 demethylation. Conditions which promote oxysterol intermediate accumulation during the demethylation process," Journal of Biological Chemistry, vol. 261, no. 36, pp. 16937-16942, 1986.

[26] D. A. Leonard, M. A. Kotarski, J. E. Tessiatore, M. F. Favata, and J. M. Trzaskos, "Post-transcriptional regulation of 3hydroxy-3-methylglutaryl coenzyme A reductase by 3 betahydroxy-lanost-8-en-32-al, an intermediate in the conversion of lanosterol to cholesterol," Archives of Biochemistry and Biophysics, vol. 310, pp. 152-157, 1994.

[27] J. M. Trzaskos, R. L. Magolda, M. F. Favata et al., "Modulation of 3-hydroxy-3-methylglutaryl-CoA reductase by $15 \alpha$ fluorolanost-7-en-3 $\beta$-ol. A mechanism-based inhibitor of cholesterol biosynthesis," Journal of Biological Chemistry, vol. 268, no. 30, pp. 22591-22599, 1993.

[28] G. C. Ness, D. Lopez, C. M. Chambers et al., "Effects of 15Oxa-32-vinyl-lanost-8-ene-3 $\beta, 32$ diol on the expression of 3hydroxy-3-methylglutaryl coenzyme a reductase and low density lipoprotein receptor in rat liver," Archives of Biochemistry and Biophysics, vol. 357, no. 2, pp. 259-264, 1998.

[29] C. Murphy, H. Ledmyr, E. Ehrenborg, and M. Gafvels, "Promoter analysis of the murine squalene epoxidase gene. Identification of a 205 bp homing region regulated by both SREBP'S and NF-Y," Biochimica et Biophysica Acta, vol. 1761, pp. 12131227, 2006.

[30] D. Rozman, M. Fink, G. M. Fimia, P. Sassone-Corsi, and M. R. Waterman, "Cyclic adenosine 3',5'- Monophosphate(cAMP)/cAMP-responsive element modulator (CREM)dependent regulation of cholesterogenic lanosterol $14 \alpha$ demethylase (CYP51) in spermatids," Molecular Endocrinology, vol. 13, no. 11, pp. 1951-1962, 1999.

[31] D. Lopez, C. M. Chambers, and G. C. Ness, "3-hydroxy3-methylglutaryl coenzyme A reductase inhibitors unmask cryptic regulatory mechanisms," Archives of Biochemistry and Biophysics, vol. 343, no. 1, pp. 118-122, 1997.

[32] C. M. Chambers and G. C. Ness, "Translational regulation of hepatic HMG-CoA reductase by dietary cholesterol," Biochemical and Biophysical Research Communications, vol. 232, no. 2, pp. 278-281, 1997.

[33] G. C. Ness, K. R. Gertz, and R. C. Holland, "Regulation of hepatic lanosterol 14 alpha-demethylase gene expression by dietary cholesterol and cholesterol-lowering agents," Archives of Biochemistry and Biophysics, vol. 395, pp. 233-238, 2001.

[34] W. Chen, K. Suruga, N. Nishimura, T. Gouda, V. N. Lam, and H. Yokogoshi, "Comparative regulation of major enzymes in the bile acid biosynthesis pathway by cholesterol, cholate and taurine in mice and rats," Life Sciences, vol. 77, no. 7, pp. 746757, 2005.

[35] S. K. Cheema, D. Cikaluk, and L. B. Agellon, "Dietary fats modulate the regulatory potential of dietary cholesterol on cholesterol $7 \alpha$-hydroxylase gene expression," Journal of Lipid Research, vol. 38, no. 2, pp. 315-323, 1997.

[36] S. Dueland, J. Drisko, L. Graf, D. Machleder, A. J. Lusis, and R. A. Davis, "Effect of dietary cholesterol and taurocholate on cholesterol $7 \alpha$-hydroxylase and hepatic LDL receptors in inbred mice," Journal of Lipid Research, vol. 34, no. 6, pp. 923931, 1993.

[37] Z. Zhang, H. Wang, R. Jiao et al., "Choosing hamsters but not rats as a model for studying plasma cholesterol-lowering activity of functional foods," Molecular Nutrition and Food Research, vol. 53, no. 7, pp. 921-930, 2009. 
[38] F. Legendre, P. Bogdanowicz, K. Boumediene, and J. P. Pujol, "Role of interleukin 6 (IL-6)/IL-6R-induced signal tranducers and activators of transcription and mitogen-activated protein kinase/extracellular," Journal of Rheumatology, vol. 32, no. 7, pp. 1307-1316, 2005.

[39] K. Rutkute and M. N. Nikolova-Karakashian, "Regulation of insulin-like growth factor binding protein-1 expression during aging," Biochemical and Biophysical Research Communications, vol. 361, no. 2, pp. 263-269, 2007.

[40] M. Wallander, A. Norhammar, K. Malmberg, J. Öhrvik, L. Rydén, and K. Brismar, "IGF binding protein 1 predicts cardiovascular morbidity and mortality in patients with acute myocardial infarction and type 2 diabetes," Diabetes Care, vol. 30, no. 9, pp. 2343-2348, 2007.

[41] A. Borai, C. Livingstone, and G. A. A. Ferns, "The biochemical assessment of insulin resistance," Annals of Clinical Biochemistry, vol. 44, no. 4, pp. 324-342, 2007.

[42] F. Zhang, K. Sjoholm, and Q. Zhang, "Attenuation of insulin secretion by insulin-like growth factor binding protein-1 in pancreatic beta-cells," Biochemical and Biophysical Research Communications, vol. 362, pp. 152-157, 2007.

[43] B. M. Wolpin, D. S. Michaud, E. L. Giovannucci et al., "Circulating insulin-like growth factor binding protein-1 and the risk of pancreatic cancer," Cancer Research, vol. 67, no. 16, pp. 7923-7928, 2007. 

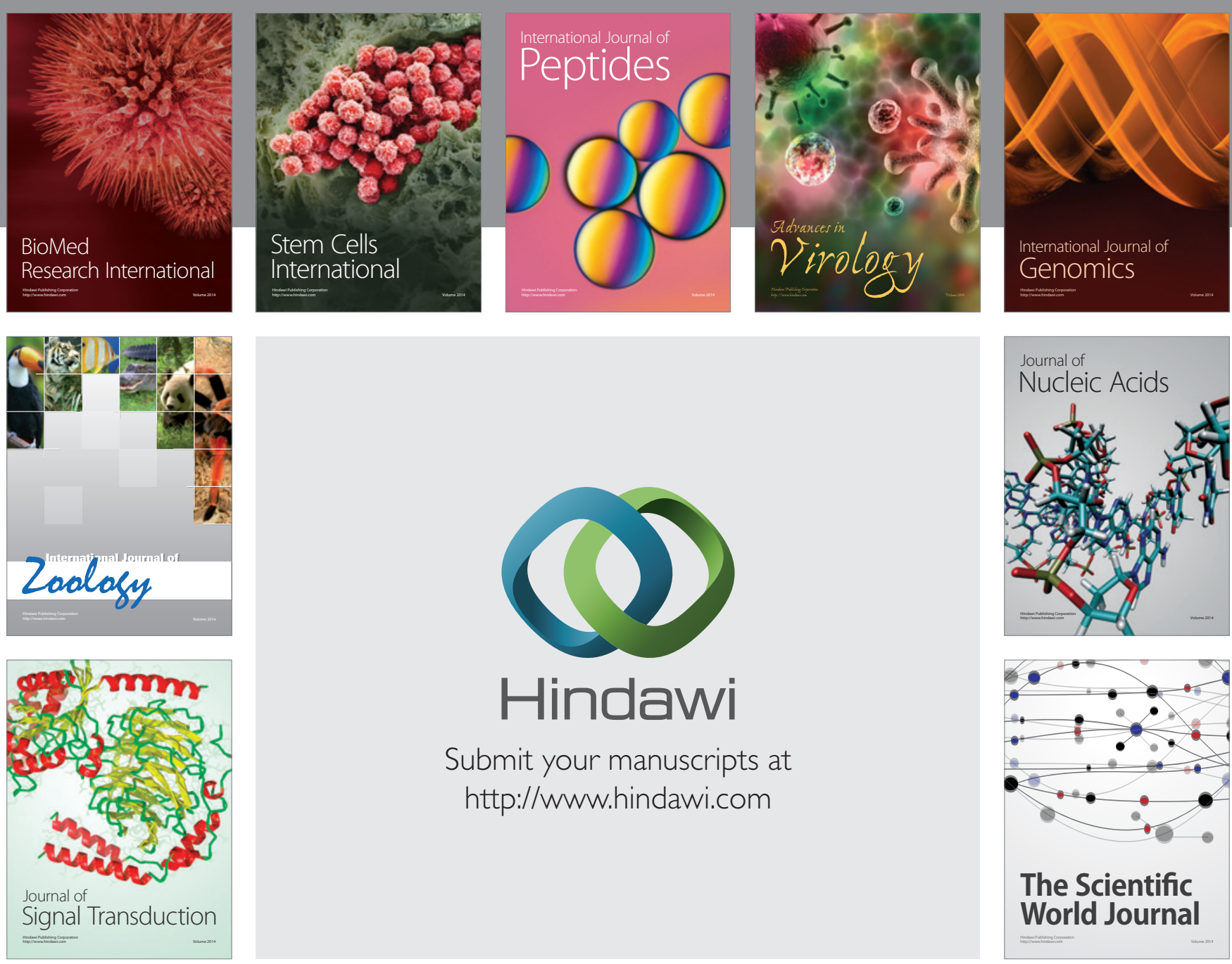

Submit your manuscripts at

http://www.hindawi.com
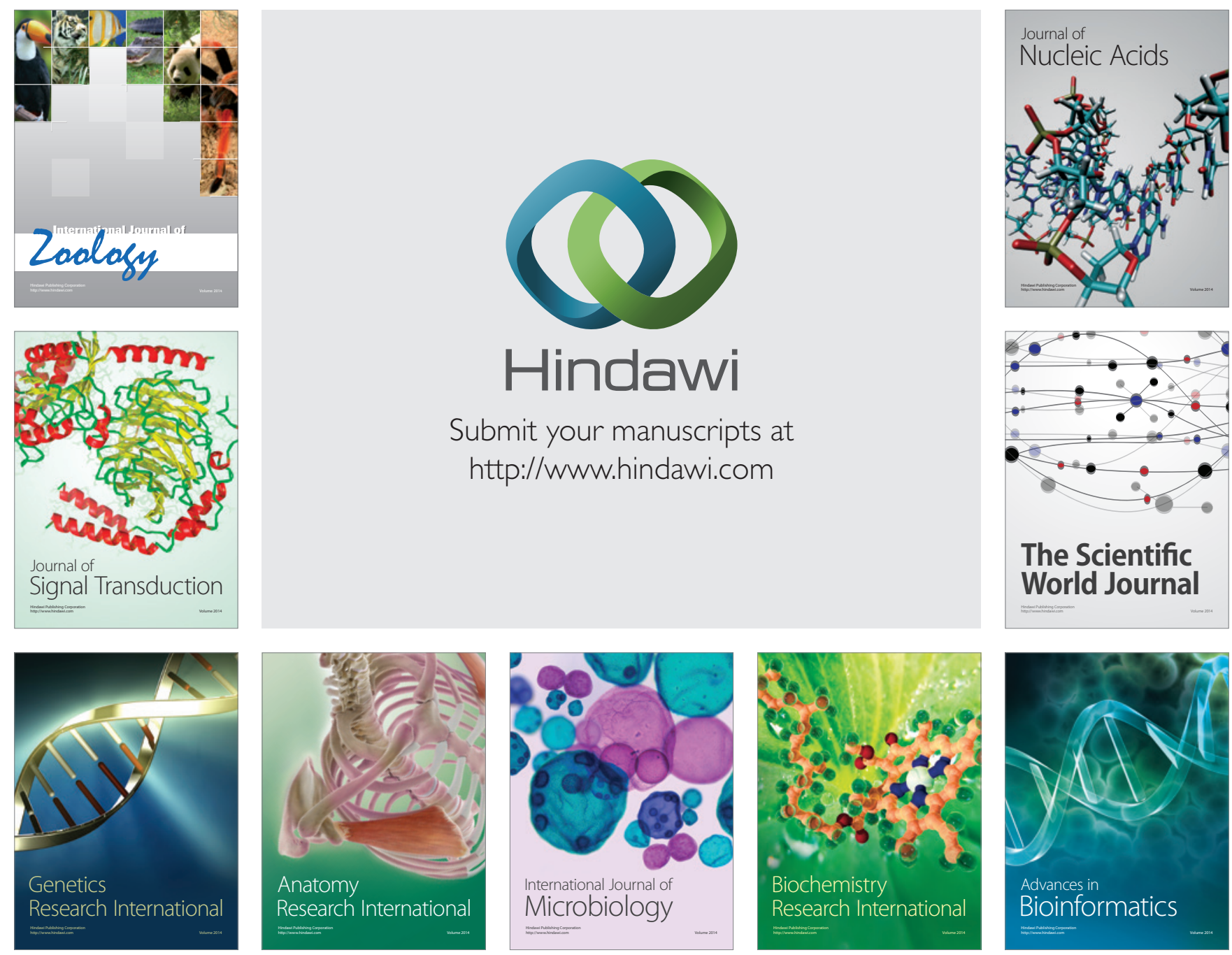

The Scientific World Journal
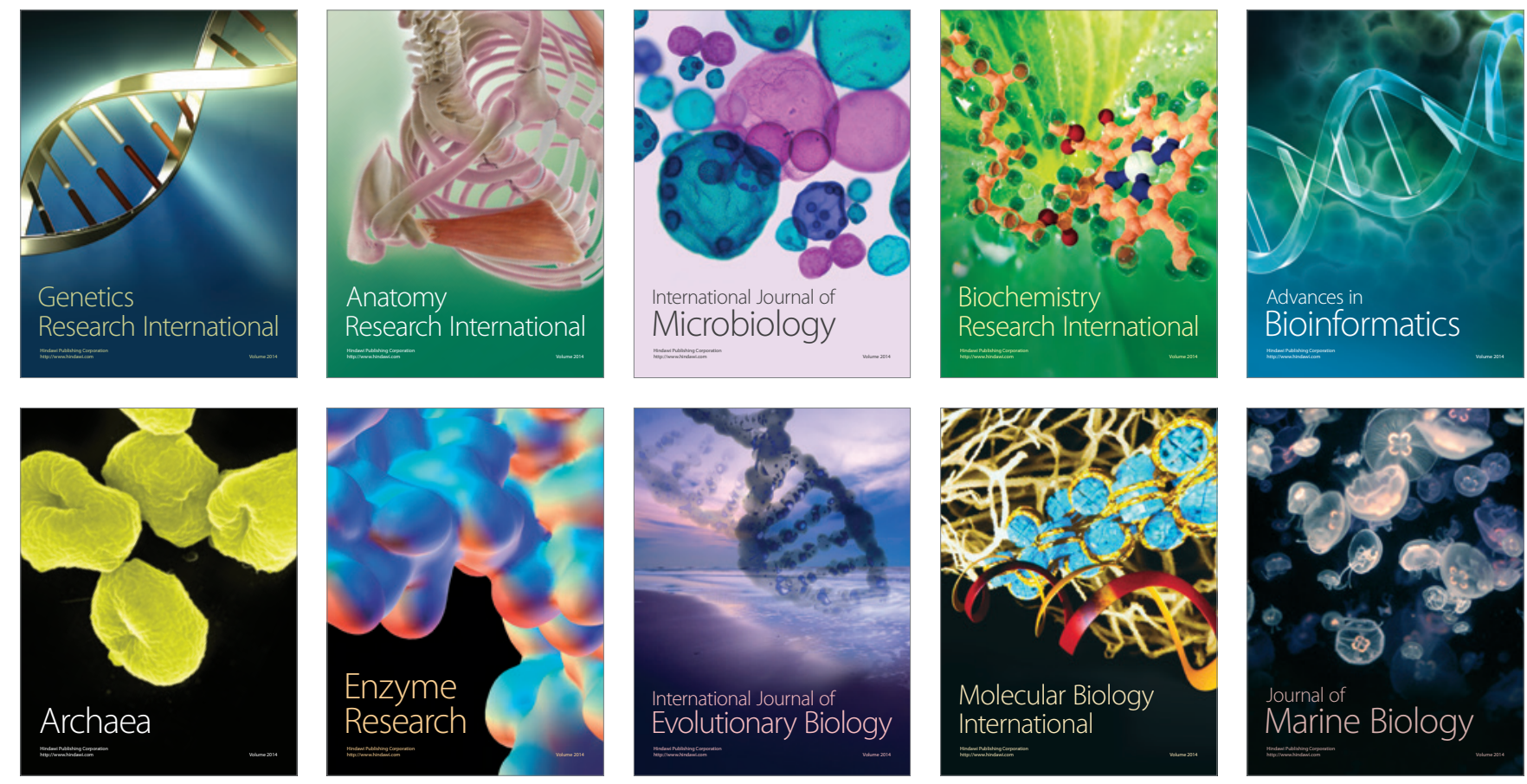